\title{
Adhesion and friction of a multiwalled carbon nanotube sliding against single-walled carbon nanotube
}

\author{
Bharat Bhushan, ${ }^{1, *}$ Xing Ling, ${ }^{1}$ Alain Jungen, ${ }^{2}$ and Christofer Hierold ${ }^{2}$ \\ ${ }^{1}$ Nanotribology Laboratory for Information Storage and MEMS/NEMS (NLIM), The Ohio State University, \\ 201 W. 19th Avenue, Columbus, Ohio 43210-1142, USA \\ ${ }^{2}$ Micro and Nanosystems, ETH Zurich, Tannenstrasse 3, CH-8092 Zurich, Switzerland \\ (Received 30 January 2008; revised manuscript received 27 March 2008; published 29 April 2008)
}

\begin{abstract}
The adhesion and friction at crossed nanotube junctions were investigated in ambient by using an atomic force microscope (AFM) in tapping mode. A multiwalled carbon nanotube (MWNT) tip attached to a conventional AFM probe was scanned across a single-walled carbon nanotube (SWNT) suspended over a 2- $\mu$ m-wide trench. The interaction between nanotubes was found to critically depend on the morphology of the MWNT tip, which was determined from force-distance curves and scans performed against hard trench surface. The interaction between nanotubes caused the attenuation of vibrational amplitude of AFM cantilever, from which the frictional force between nanotubes was obtained by analyzing the dissipated vibrational power of cantilever. The adhesive force between nanotubes was measured from the vertical cantilever deflection when the MWNT tip end detached from the shell of the SWNT. From the friction and adhesion data, an experimental value of coefficient of friction of $0.006 \pm 0.003$ is obtained for the sliding between nanotubes, which is comparable to the reported value of graphite on nanoscale. The shear strength between nanotubes is derived to be $4 \pm 1 \mathrm{MPa}$ by using a continuum model, which is in accordance with the value of $2 \mathrm{MPa}$ reported for the sliding of MWNT on graphite in ambient. It is nearly 2 orders of magnitude larger than the interlayer shear strength of 0.05 MPa reported for MWNT in vacuum. We attribute the difference between the intertube and the interlayer frictions to the presence of water at the nanotube-nanotube interface in ambient.
\end{abstract}

DOI: 10.1103/PhysRevB.77.165428

PACS number(s): 68.35.Np, 46.55.+d, 61.48.De, 07.79.Lh

\section{INTRODUCTION}

Carbon nanotubes (CNTs), since their discovery in 1991 by Iijima, ${ }^{1}$ have attracted intensive research activities due to their remarkable mechanical and electrical properties., ${ }^{2,3}$ Formed by curling graphene sheets into nanometer-diameter tubes, CNTs inherit the high stiffness and high strength of the basal plane of graphite while preserving its low density. ${ }^{4-8}$ With their tubular structure and graphitelike $s p^{2}$ bonds, CNTs can sustain repetitive large deformation without catastrophic failure. ${ }^{9,10}$ Their electronic properties can vary from metallic to semiconducting by changing their diameter and chirality. ${ }^{11}$ Attempts have been made to develop reinforced polymeric composites, ${ }^{12}$ ultrasharp resilient nanoprobes ${ }^{13}$ capable of chemical and biological discrimination, ${ }^{14}$ nanoscale electromechanical devices, ${ }^{15,16}$ and molecular electronics ${ }^{17-20}$ based on CNTs. The performance of composites used in mechanical devices critically relies on the mechanical and tribological properties of CNTs, ${ }^{21,22}$ including the static and dynamic tube matrix, intertube, and interlayer friction and adhesion. The direct tribological characterization between two nanotubes is scarce. A systematic study of adhesion and friction in intertubular contacts has recently been carried out by Bhushan et al. ${ }^{23}$ While the interlayer friction properties of CNTs were broadly explored in vacuum condition envisioning their relevance to use CNTs as ideal linear and rotational nanobearings. ${ }^{24-30}$

The interlayer interaction is known to be predominantly van der Waals. ${ }^{1}$ Similar to the sliding between graphite in dry contact, ${ }^{31}$ interlayer sliding inside multiwalled carbon nanotubes (MWNTs) exhibited superlubricity due to the socalled incommensurate contact, ${ }^{32}$ bringing down the shear strength to as low as $0.05 \mathrm{MPa}$ when the shell structure was defect-free. ${ }^{24,26}$ The major energy dissipation mechanism was believed to arise from the interaction of the open end of outer tubes (where dangling bonds and defects were present) with the inner shells. ${ }^{29,33}$ Thus, the interlayer friction would be defect dependent rather than area dependent as predicted by the classic friction theory. ${ }^{34-36}$ Although incommensurate contact was also in action when manipulating a MWNT to slide on graphite surface, ${ }^{37-39}$ the frictional force between MWNT and graphite has been found to be much higher than the interlayer frictional force. Based on continuum analysis using adhesive model, the shear strength was calculated to be $2 \mathrm{MPa},{ }^{39}$ nearly 2 orders of magnitude larger than the interlayer shear strength. The discrepancy is worthy of further clarification. Stick-slip-like features had been observed on the interlayer sliding and the rolling of MWNT on graphite due to the interlayer adhesion modulated by defects ${ }^{26}$ and the commensurate contact, ${ }^{39}$ respectively. It is interesting to know if it can be observed on the sliding between two nanotubes.

In the present work, comprehensive tribological characterization between two individual nanotubes was performed with an atomic force microscopy (AFM) operating in tapping mode. One nanotube was a MWNT welded to the tip of a silicon AFM probe, and the other one was a single-walled carbon nanotube (SWNT) suspended over a microtrench. The MWNT tip was used to scan in the tapping mode over the SWNT in a crossed geometry. The interaction between the two nanotubes caused changes in the tapping amplitude and the vertical deflection of the AFM cantilever. Compared to the vertical deflection signal, the change in the tapping amplitude signal is more sensitive to the very small dissipa- 


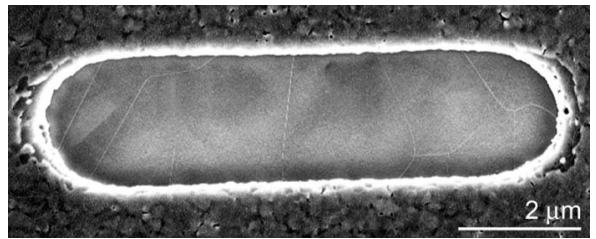

FIG. 1. SEM image of single-walled carbon nanotubes suspended on the top of a microtrench made of polycrystalline silicon.

tive frictional force between nanotubes, and the latter is used to calculate the frictional force. The tapping amplitude data during the initial contact of nanotubes are used for calculations; these data are used to avoid the influence to the friction from the normal force between nanotubes, which is expected to be small. Near the end of the scanning, the MWNT tip detached from the SWNT and the adhesive force between them caused significant changes to the vertical deflection of cantilever, which is used to calculate the adhesive force between nanotubes. By comparing the frictional force to the adhesive force, an experimental value of coefficient of friction between nanotubes is determined. By using a continuum model, the contact size and the shear stress between nanotubes are derived. The friction mechanism between nanotubes is then discussed and compared to the interlayer sliding and the sliding between MWNT and graphite.

\section{EXPERIMENT}

\section{A. Single-walled carbon nanotubes suspended on microtrench}

SWNT bridges were synthesized by catalytic thermal chemical vapor deposition (CVD) in a low pressure furnace. ${ }^{40,41}$ Prior to the CVD growth, microchips were fabricated by surface micromachining of $1.5 \mu \mathrm{m}$ thick polycrystalline silicon (poly-Si) layers. The poly-Si layers were uniformly coated with a bimetallic thin film of $8 \mathrm{~nm} \mathrm{Al}$ and $1 \mathrm{~nm} \mathrm{Ni}$ in a sputter system. The film thickness was monitored in situ by a quartz crystal microbalance. The chips were transferred in air and subjected to hydrogen pretreatment at $0.02 \mathrm{MPa}$ and $850{ }^{\circ} \mathrm{C}$ for $10 \mathrm{~min}$ to allow the reduction of nickel oxides and formation of $\mathrm{Ni}$ islands. The latter serve as catalytic seeds for the growth of SWNTs under methane and hydrogen $(3: 1)$ at $0.02 \mathrm{MPa}$ and $850{ }^{\circ} \mathrm{C}$ for 15 min. Heating and cooling were performed under vacuum, and the chamber was opened only after cooling to at least $250{ }^{\circ} \mathrm{C}$. A typical scanning electron microscopy (SEM) image of SWNTs suspended on top of a trench of $2 \mu \mathrm{m}$ wide and $1.5 \mu \mathrm{m}$ deep is shown in Fig. 1. The SWNTs appear taut and straight and some exhibit a branched structure.

\section{B. Multiwalled carbon nanotube tip and tribological measurements}

The MWNT tip was prepared by mounting an individual MWNT to the tip of a conventional AFM probe by using a micromanipulator operated under an inverted microscope. ${ }^{42}$ Low-density and individually separated MWNTs were grown by CVD on a Pt wire coated with a liquid catalyst solution. By using an inverted microscope at $500 \times$ magnification, a

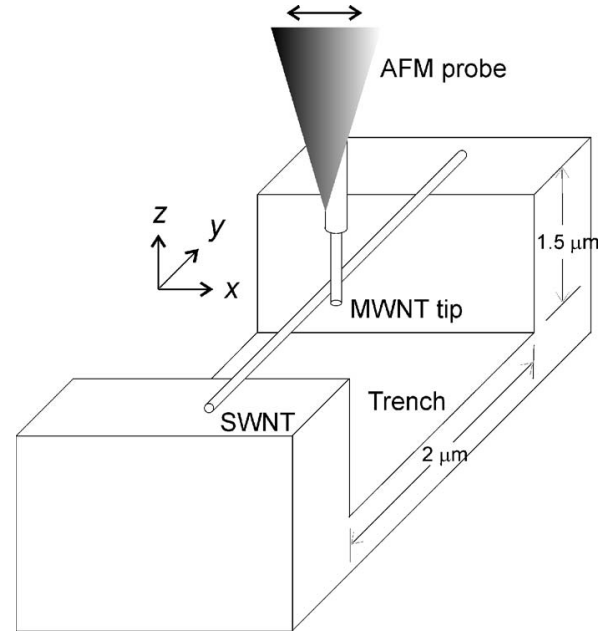

FIG. 2. Schematic of the experimental setup for tribological measurements between individual nanotubes. The double arrow indicates the fast scanning direction ( $x$ direction).

single MWNT with a typical length greater than $10 \mu \mathrm{m}$ was transferred to the tip of a Si cantilever coated with a $15 \mathrm{~nm}$ Ni film. The relative position of the MWNT to the Si tip was manually manipulated by using a pair of microtranslators. When the nanotube and the Si tip were in close proximity, an electrical potential of $1-2 \mathrm{~V}$ was applied to improve the alignment of the nanotube with respect to the apex of the $\mathrm{Si}$ tip, and then by increasing the voltage to $10 \mathrm{~V}$ and above the MWNT was detached from its source at the point of defects. The applied voltage caused local heating at the MWNT-Nicoated-Si-tip interface, which strengthens the interface via physical welding the MWNT to the Ni film and/or formation of chemical bonds between the MWNT and the Ni-coated $\mathrm{Si}$ tip. The normal spring constant $k_{z}$ of the MWNT probe has been calibrated to be $1.35 \mathrm{~N} / \mathrm{m}$ using the method proposed by Sader et $a l .^{43}$

To measure the adhesion and friction between nanotubes, the MWNT tip was scanned across SWNTs suspended on a microtrench of $2 \mu \mathrm{m}$ wide and $1.5 \mu \mathrm{m}$ deep by using an AFM (Fig. 2). As in the elastic modulus ${ }^{5}$ and strain-at-break $^{44}$ measurements of SWNT ropes, SWNTs were freely suspended across a trench to avoid adhesion between the nanotubes and an underlying substrate. The trench was aligned with the long axis of the AFM cantilever. During tapping mode imaging with an oscillating frequency of $47.5 \mathrm{KHz}$ by manipulating the center and size of scan area, the MWNT tip was positioned inside the trench and close to one of its ends. The AFM feedback was then disabled, and the probe was lifted by a predefined height between $1.2-1.5 \mu \mathrm{m}$ from the bottom of trench. Line scans and area scans with variable scan size ranging from $500 \mathrm{~nm}$ to $4 \mu \mathrm{m}$ were performed. The scan angle and scan rate were fixed to zero (fast scanning direction is parallel to the long axis of cantilever, Fig. 2) and $1 \mathrm{~Hz}$, respectively, and the height of the probe was kept constant during the scans by disabling the AFM feedback response. The center of the scan was modulated along the long axis of the trench in small steps (normally $10 \%$ of the scan size) in order to locate suspended SWNTs. The scan process was repeated many times until 
suspended SWNTs bridging the trench were found. During scans across suspended SWNTs, the interaction between the MWNT tip and the SWNTs caused changes in the tapping cantilever amplitude and vertical deflection signals, from which the friction and adhesion between nanotubes can be obtained using the strategies developed in Sec. III.

Between some scans, we measured force-distance curves by oscillating the MWNT tip against the trench (bottom or top), during which cantilever amplitude and deflection were recorded as a function of the distance between the MWNT probe and the trench surface. The force curves were used to evaluate the morphologies of the MWNT tip, which could change with scans. The temperature and relative humidity were $21 \pm 1{ }^{\circ} \mathrm{C}$ and $30 \pm 5 \%$, respectively.

\section{RESULTS AND DISCUSSION}

We will first examine the force-distance curves and the scans of MWNT tip against hard tube support layer and their correlation with the change in the morphology of the MWNT tip during scanning. The sliding between nanotubes was then explored with the end of the MWNT tip $\sim 100 \mathrm{~nm}$ below the suspended SWNT and at the same height as the SWNT. The attenuation in tapping amplitude during the initial contact between two nanotubes was used to evaluate the friction, while the cantilever vertical deflection signal generated when nanotubes detached from each other was used to evaluate the adhesive force between nanotubes. Based on the measured friction and adhesion, the experimental value of coefficient of friction and the shear strength between nanotubes were derived and compared to the values reported for interlayer sliding and the sliding between MWNT and graphite. Lastly, the friction mechanism between two nanotubes is discussed.

\section{A. Force-distance curves and scan of multiwalled carbon nanotube tip on tube support layer and their correlation with the change in the tip morphology}

The interaction of a MWNT tip with a SWNT suspended on a trench was found to depend critically on the morphology of the MWNT tip, which changed during repetitive scans inside the trench. During the initial scanning cycles, the MWNT tip behaved as a stiff rod. With a preset height of tip end lower than the tube support layer by several tens of nanometers or more, scans on the tube support layer (feedback was turned off as described in the experimental section) immediately reduced the cantilever amplitude to zero and increased the cantilever deflection by nearly the height difference between the tip end and the tube support layer, and scans across suspended SWNTs always led to broken SWNTs or dragging of SWNTs along the tube support layer indicated by events such as discontinued scan lines in area scans or unrepeatable saw tooth pattern in line scans. This situation was undesirable since in the experimental scheme mentioned above it is necessary for the MWNT tip to slide across the SWNT to study the sliding friction between nanotubes. After many scans, the MWNT tip became more compliant, possibly due to partial stripping of its outer shells, which is desirable to minimize damage to SWNTs. We first noticed this change during normal tapping mode imaging (feedback on) inside the trench or on the tube support layer. The imaging became quite unstable, and trace and retrace scan lines showed large discrepancy and hysteresis. However, accompanying this change, the MWNT tip was able to scan across the suspended SWNT, enabling the examination of sliding behavior between two nanotubes.

\section{Force-distance curves}

In order to determine the morphology of the tip, forcedistance curves were obtained in tapping mode by oscillating the MWNT tip against the trench surface during the scanning cycles. In Figs. 3(a) and 3(b), force-distance curves during the first cycle, during the test, and after $\sim 500$ scanning cycles (estimated from the summation of both line scans and area scans performed) are displayed with corresponding SEM images and/or schematics [Fig. 3(c)] for each tip morphology. The diameter of the MWNT tip is measured from SEM images to be about $100 \mathrm{~nm}$. During the first cycle, its length extending beyond the silicon tip is $1.65 \mu \mathrm{m}$ [Fig. 3(c), top]. From the amplitude curves [Fig. 3(a), top], we observe a rapid decline of cantilever amplitude to zero after the contact of the MWNT tip with the trench surface, followed by the linear increase of cantilever deflection until the buckling point [Fig. 3(b), top], defined in the approaching curve when the deflection started to deviate from the linear increase. The behavior of the MWNT tip before the buckling point resembles a normal probe (incompressible), ${ }^{45}$ as expected for a 100-nm-diameter MWNT. $^{7}$ The buckling force measured from the top deflection curve is $159 \mathrm{nN}$, much smaller than the Euler buckling force of $17.8 \mu \mathrm{N}$ calculated by using ${ }^{46}$

$$
F_{\text {Euler }}=\frac{\pi^{2} E I}{l^{2}}
$$

where $E$ is Young's modulus that is assumed to be $1 \mathrm{TPa},{ }^{7}$ $I \approx \pi r^{4} / 4$, is the area moment of inertia, $r$ is the outer radius of MWNT equaling to $50 \mathrm{~nm}$ here and the inner radius of MWNT is assumed to be zero, and $l$ is the length of the MWNT tip of $1.65 \mu \mathrm{m}$ measured from SEM images. Even if the MWNT has only one shell, the calculated Euler buckling force of $479 \mathrm{nN}$ using an inner radius of $49.66 \mathrm{~nm}$, as obtained by subtracting the outer radius with the thickness of one MWNT shell of $0.34 \mathrm{~nm},{ }^{1}$ is still larger than the measured value. The failure of the Euler buckling model was recently addressed by Yap et $a l .{ }^{47}$ They proposed a symmetrical shell buckling mode to account for the small buckling force and the negative stiffness in force-distance curve after buckling. We noticed in side-view SEM images the MWNT tip is not exactly $90^{\circ}$ below the AFM cantilever. The inclination of the nanotube in AFM can be evaluated by comparing the length measured from force-distance curves (the distance between the first and the second linear slopes in the approach curve) with the length in SEM. It is about $48^{\circ}$ away from the vertical direction ( $z$ direction). After scanning cycles, the MWNT tip is shortened to $0.61 \mu \mathrm{m}$ [Fig. 3(c), bottom], which indicates the MWNT tip was either fractured or retracted along the silicon tip. Its force-distance curves [Figs. 3(a) and 3(b), bottom] were basically the same as be- 


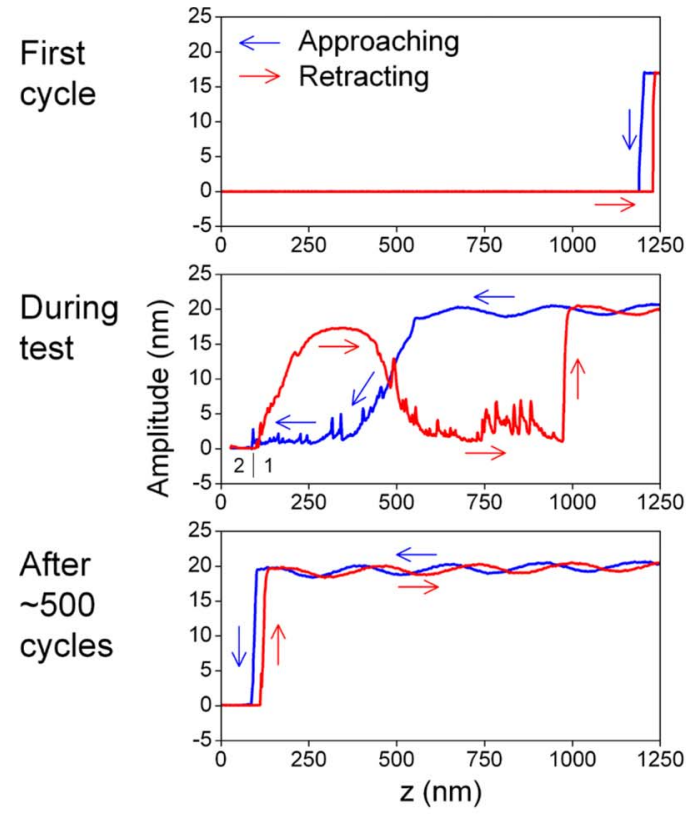

(a) Tapping amplitude
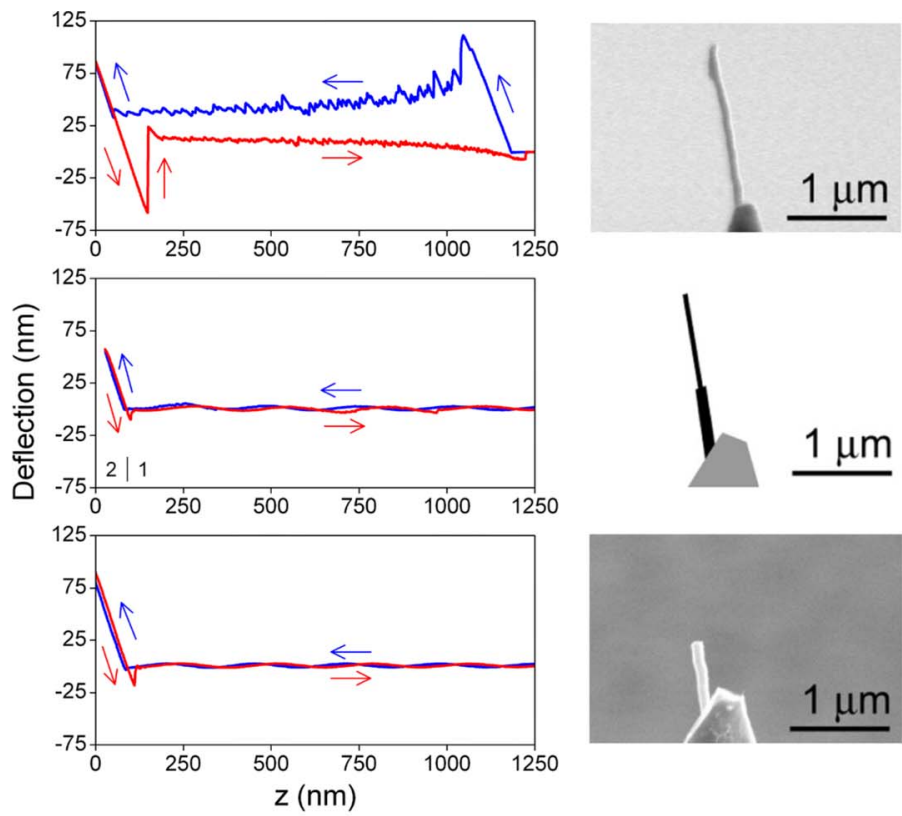

(b) Vertical deflection
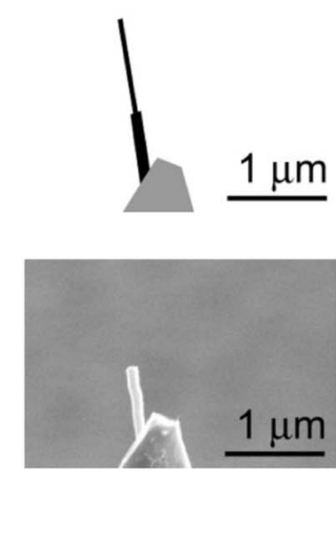

(c) SEM/Schematic

FIG. 3. (Color online) (a) Cantilever tapping amplitude and (b) vertical deflection signals (representative of at least 10 cycles reproducible within 5\%) as a function of distance, and (c) SEM/schematic images of the multiwalled carbon nanotube tip during the first cycle, during the test, and after $\sim 500$ cycles. The SEM image for the tip during the test was not taken; instead, a tip schematic is drawn. At the tip end, some shells of the MWNT were stripped off (refer to the text for details), leaving a thin tube with smaller diameter extended from a thick base.

fore the scanning cycles, except the deflection curve did not display buckling in the load range we explored.

The force-distance curves measured during the test show distinctive features [Figs. 3(a) and 3(b) middle]. During approach, to reduce the amplitude from $18.8 \mathrm{~nm}$ (free amplitude) to $1.9 \mathrm{~nm}$, it took the MWNT probe an approaching distance of $180 \mathrm{~nm}$ from the beginning of the downward slope [Fig. 3(a), middle] instead of $16.9 \mathrm{~nm}$ as expected for a normal probe. The small yet nonzero amplitude of $1.9 \mathrm{~nm}$ held for an additional approaching distance of $288 \mathrm{~nm}$ then jumped to zero, followed by rapid linear increase of cantilever deflection. To facilitate the description and analysis of the force-distance curves, we divided them into two regions at the point cantilever deflection started to increase [Figs. 3(a) and 3(b), middle]. To its right side, the region is referred as region 1 and to its left region 2. During retracting, the retracting curves repeated the approaching curves in region 2 , but in region 1 , they were different. For the retracting amplitude curve, it started to increase upon entering region 1 and reached the maximum value of $17.3 \mathrm{~nm}$ for a retracting distance of $253 \mathrm{~nm}$ from the boundary of region 1, while the approaching amplitude curve showed a platform around $1.9 \mathrm{~nm}$. Then, the retracting amplitude curve continuously decreased until $1.8 \mathrm{~nm}$ for an additional retracting distance of $271 \mathrm{~nm}$, while the approaching amplitude curve was increasing. The small yet nonzero amplitude of $1.8 \mathrm{~nm}$ held for an additional retracting distance of $360 \mathrm{~nm}$ followed by an abrupt leap to free amplitude, indicating a complete break away of the MWNT tip from the trench surface, while the approaching amplitude curve was $18.8 \mathrm{~nm}$ (free amplitude). The extraordinarily long approaching distance of $468 \mathrm{~nm}$ re- quired to fully reduce the cantilever amplitude to zero suggests the flexibility of the MWNT tip is extremely high so that even the tapping force could bend the tip. We hypothesize the increased flexibility resulted from partial stripping of the outer shells of the original MWNT during repetitive scans inside the $1.5-\mu \mathrm{m}$-deep trench prior to locating SWNTs, leaving a thin tip with smaller diameter and fewer shells extending from a thick base with a diameter of original MWNT due to the so-called sword-in-sheath breaking mechanism. ${ }^{8}$ The effect of the stripping of the shells on an increase of MWNT flexibility can be evaluated by calculating the bending stiffness (flexural modulus) of the MWNT. The bending stiffness for the MWNT tip is given by ${ }^{46}$

$$
k_{\mathrm{NT}}=\frac{3 \pi E r^{4}}{4 l^{3}} .
$$

Assuming the inner radius of the MWNT is zero, the bending stiffness is calculated to be $3.28 \mathrm{~N} / \mathrm{m}$ for the $1.65-\mu \mathrm{m}$-long MWNT tip. If the MWNT has only one shell, the bending stiffness is reduced to $88.3 \mathrm{mN} / \mathrm{m}$. It is only $2.69 \%$ of the bending stiffness of a solid MWNT. The calculation confirms shell stripping as a possible mechanism leading to extremely high flexibility of MWNT observed in force-distance curves.

By using a thin tip extending from a thick base as the tip model, the force curves shown in the middle of Figs. 3(a) and 3(b) can be explained. In region 2, the linear variation of deflection during approaching and retracting and the small pull-off force during retracting [Fig. 3(b), middle] showed no significant difference from the shortened MWNT tip [Fig. 3(b), bottom], reflecting the interaction between the thick 
base and the trench surface, which will not be discussed further here. The complexity observed in region 1 [Fig. 3(a) and 3(b), middle] can be qualitatively explained by comparing the thin MWNT tip to a spring attached to a box, sliding on the trench surface under external force applied through the free end of the spring. For the MWNT tip end (the box) to slide on the surface, the friction between them needs to be overcome. Therefore, during approaching, the MWNT spring was first compressed until the stress built inside surpassed the friction between the tip end and the surface. Then, the tip end started to slide on the surface while maintaining the compressed geometry of the MWNT spring and thus the cantilever amplitude, until the contact of the thick MWNT base with the trench surface (region 2). During retracting, the compressive stress inside the MWNT spring was released first. The amplitude consequently increased and reached the maximum when the MWNT spring was fully unloaded. When retracted further, the MWNT began to acquire tensile stress, and the amplitude reduced accordingly. The unloading and loading of the MWNT spring led to the formation of an arc in the beginning of retracting amplitude curve [Fig. 3(a), middle]. When the tensile stress inside the MWNT spring surpassed the friction between the tip end and the surface in the retracting direction, the tip end started to slide on the surface, and the accumulation of tensile stress stopped, leading to constant and small cantilever amplitude (variation due to surface roughness could occur) until the tip end detached from the surface. The MWNT tip should be fully stretched in the $z$ direction at the moment of detaching due to its high flexibility. From the force-distance curves, the length of the MWNT tip is $884 \mathrm{~nm}$, which correlates well, within experimental errors, with the length difference of $1.04 \mu \mathrm{m}$ evaluated from SEM images [Fig. 3(c), top and bottom]. Moreover, by comparing the approaching distance with the retracting distance in region 1 [Fig. 3(a), middle], the tilting angle of the MWNT tip was calculated to be $58^{\circ}$, which is in accordance with the value of $48^{\circ}$ estimated before. The consistency of the length and angle of MWNT tip obtained by using different methods quantifies the use of a thin tip extending from a thick base as the tip model for the MWNT tip during test. The tip model provides a satisfying explanation for the complex force-distance curves observed during test.

\section{Scan of multiwalled carbon nanotube tip on tube support layer}

It is also interesting to examine the scanning behavior of the stripped MWNT tip on the hard tube support layer, which helps to understand the interaction between the MWNT tip and the suspended SWNTs to be addressed. A typical scanning result of the stripped MWNT tip over the trench with its tip end of $\sim 100 \mathrm{~nm}$ below the tube support layer is shown in Fig. 4 . The scan size is $4 \mu \mathrm{m}$, wide enough to cover part of the tube support layer and part of the trench (Fig. 4, schematic). At present, we focus on the cantilever response during the scanning on the tube support layer. Note that the cantilever tapping amplitude [Fig. 4(a)] and vertical deflection [Fig. 4(b)] signals were measured from separated scans because of limited data channels available. Slight drifts of the scanner in the vertical and lateral directions between scans were observed in our experiments. The variation of cantilever deflection during entire scan was small, on the order of $1 \mathrm{~nm}$, even when the tip was scanning on the tube support layer [Fig. 4(b)]. This provided additional evidence that the stripped MWNT tip was extremely flexible and adsorbed most deformation when scanning on hard surfaces. The variation of cantilever amplitude appears to follow two distinct patterns [Fig. 4(a)]. We use the trace amplitude curve from one representative scan line as an example [profile below the trace amplitude image in Fig. 4(a)]. From its magnified view in Fig. 5(b), we observe that the amplitude curve to the right displays a vibrational pattern in the range of $8.3 \pm 2.7 \mathrm{~nm}$, where the MWNT tip should slide on the tube support layer in a steady motion. To its left, the amplitude curve forms a nearly symmetric arc with a width of $1180 \mathrm{~nm}$. The height of the arc is $20.0 \mathrm{~nm}$, which is close to the free vibrational amplitude of the cantilever [profile in Fig. 4(a)].

To explain the formation of such an arc, we mark three points $A_{T}, B_{T}$, and $C_{T}$ on the arc [Fig. 5(b)] and illustrate the possible contact geometries of the MWNT tip on the tube support layer for the three points in the schematic of Fig. 5(b). Note that the points in Fig. 5 are only indications of specific lateral displacements of AFM probe, $x$ values, and are not tied to a specific curve. The difference between the arc and the vibrational pattern due to the sliding of the MWNT tip on the tube support layer suggests that the MWNT tip end should be fixed in region $A_{T}-C_{T}$ (no sliding). Moreover, based on the observed symmetries of the trace and retrace arcs [Figs. 5(b) and 4(a)], the MWNT tip end should be straight below the MWNT tip base when the MWNT probe had moved to the arc top at point $B_{T}$. At point $A_{T}$, the MWNT tip was tensile strained due to the friction between the MWNT tip and the tube support layer at the end of previous retrace scan. The strain is negligible as the elastic modulus of the nanotube is very high; upon the displacement of the MWNT probe in the trace direction, the strain rapidly released, producing a steep arc slope. Since at point $A_{T}$, the MWNT tip was fully stretched and its tip end located at point $B_{T}$ using the afore-obtained length of the MWNT tip of $884 \mathrm{~nm}$ and the measured distance of $A_{T}-B_{T}$ of $590 \mathrm{~nm}$, the height of the MWNT tip base with respect to the tube support layer is calculated to be $658 \mathrm{~nm}$, indicating the MWNT tip end was $226 \mathrm{~nm}$ below the tube support layer if the MWNT tip was aligned in the $z$ direction. The discrepancy between the value of $226 \mathrm{~nm}$ and the experimentally set value of $100 \mathrm{~nm}$ is accounted for by introducing a tilting angle of $31^{\circ}$ to the MWNT tip with respect to the $z$ direction. The calculation agrees well, within experimental errors, with the results obtained before from SEM images and forcedistance curves.

Upon further trace displacement, the MWNT was gradually compressed and bent, and the compressive stress reached the maximum at point $B_{T}$. Notably at point $B_{T}$, where the MWNT tip was mostly compressed, the cantilever amplitude is increased to a local maximum. Such a counterintuitive phenomenon can be explained by treating the bent MWNT tip as a preloaded (compressed) spring. When the spring is tilted by an angle of $\theta$ with respect to the $z$ direction, its effective spring constant is increased approximately 


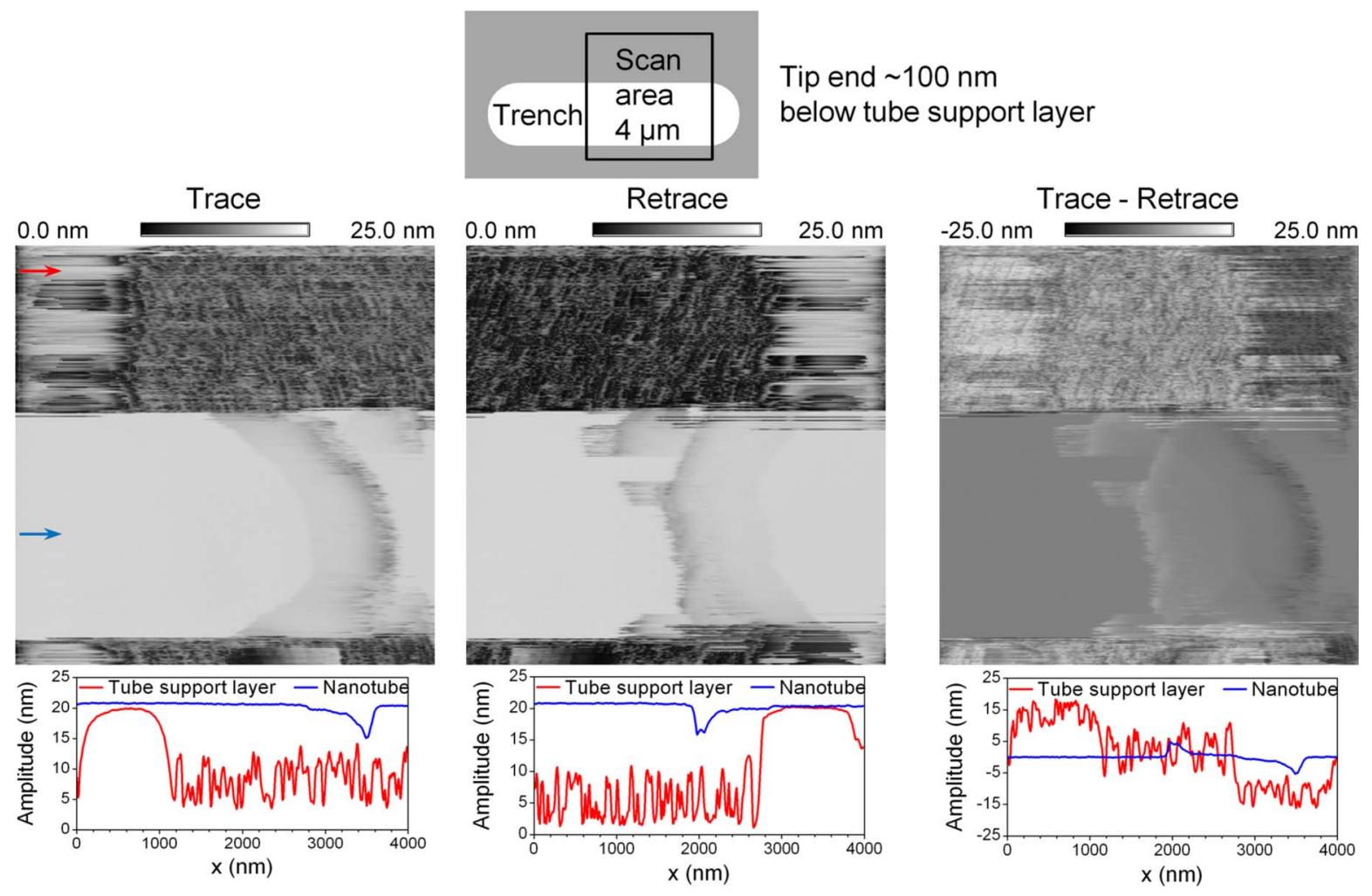

(a) Tapping amplitude
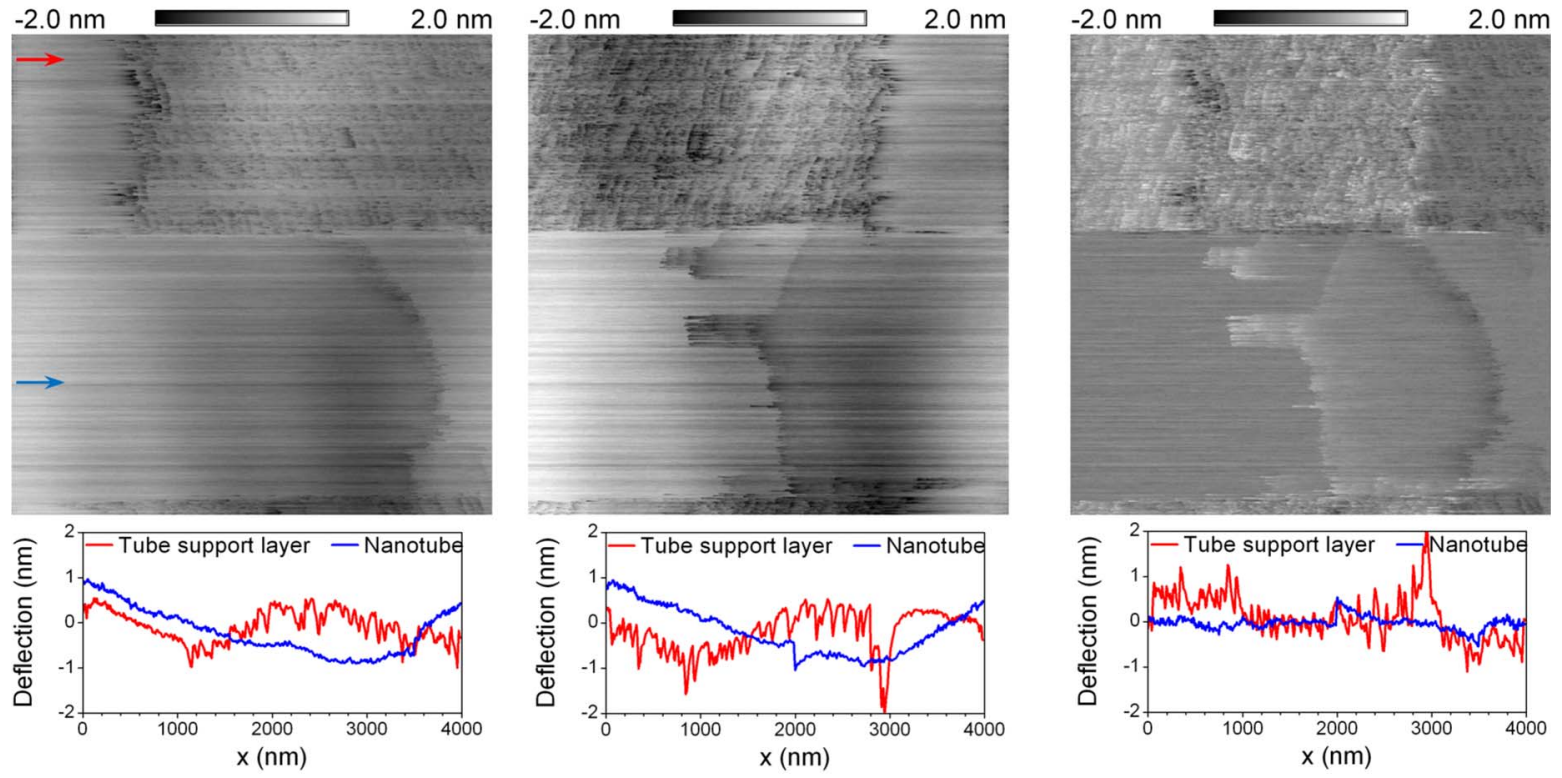

(b) Vertical deflection

FIG. 4. (Color online) From left to right, the images correspond to (a) cantilever tapping amplitude signals and (b) cantilever vertical deflection recorded during the trace and retrace scans and their difference. The deflection was captured on a separate scan immediately after the capture of amplitude. Profiles are depicted below each image for the scan lines marked by the arrows. The scan lines marked by the upper arrows are on the tube support layer. The scan lines marked by the lower arrows are through the suspended SWNT. The scan size is $4 \mu \mathrm{m}$ and the tip end is about $100 \mathrm{~nm}$ lower than the tube support layer. The position of the scan area relative to the trench is depicted in the top schematic.

by a factor of $1 / \cos ^{2} \theta$. Therefore, in straight configuration (at point $B_{T}$ ), the spring has the smallest effective spring constant. The presence of the MWNT spring increased the resonance frequency of cantilever and consequently reduced the amplitude of cantilever, which was vibrated under its natural resonance frequency. At point $B_{T}$, the smallest effective spring constant resulted in the smallest offset of resonance frequency, leading to the maximum cantilever ampli- 

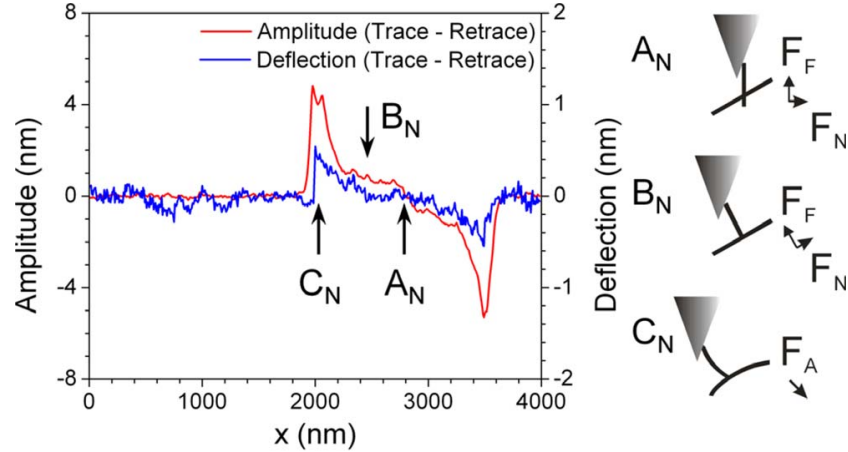

(a) Nanotube

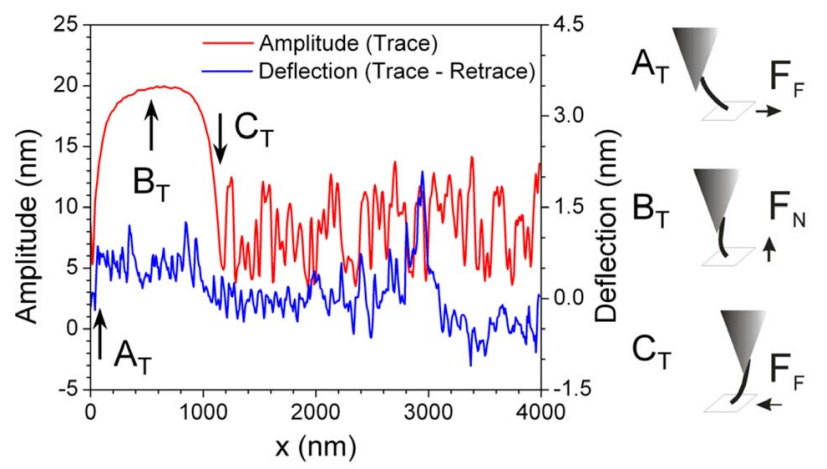

(b) Tube support layer

FIG. 5. (Color online) Cantilever tapping amplitude and vertical deflection signals extracted from the scan lines through the suspended SWNT (a) and on the tube support layer (b) from Fig. 4. The MWNT tip contact geometries and forces corresponding to three points for SWNT $\left(A_{N}, B_{N}\right.$, and $\left.C_{N}\right)$ and tube support layer $\left(A_{T}\right.$, $B_{T}$, and $C_{T}$ ) are illustrated to the right. The tapping amplitude signal measured at point $A_{N}$ for each scan lines is used to calculate the frictional force between nanotubes while the vertical deflection signal measured at point $C_{N}$ is used for the calculation of the adhesion force between nanotubes.

tude compared to other points where the MWNT spring was tilted. Besides the elastic deformation of the MWNT tip, additional amplitude attenuation could occur due to the energy dissipation between the MWNT tip and the tube support layer, especially when the MWNT was subjected to the tensile stress during tip sliding on the substrate. This explains the formation of steep arc slopes.

With the MWNT tip end still fixed at point $B_{T}$, the compressive stress inside the MWNT tip is gradually released when the MWNT tip base is displaced from point $B_{T}$ to $C_{T}$ and turned into tensile stress at the edge of $C_{T}$ due to the friction between the MWNT tip end and the tube support layer in the retrace direction. When the tensile stress overcame the friction, the MWNT tip end started to slide at point $C_{T}$. After point $C_{T}$, the friction modulated by the surface roughness caused the vibrational pattern in the amplitude curve as mentioned before.

Now, we look at the vertical deflection difference curve shown in Fig. 5(b). In region $A_{T} C_{T}$, the trace deflection caused by the repulsive force between the MWNT tip and the tube support layer should be nearly zero since the amplitude arc is close to the free vibrational amplitude of cantilever. Therefore, the difference between the trace deflection and the retrace deflection here of about $0.6 \pm 0.2 \mathrm{~nm}$ should primarily reflect the magnitude of friction experienced during the retrace scan [Fig. 4(b)]. Although the friction changed its direction with the scan direction, the friction induced deflection, however, remained nearly constant regardless of the scan directions [Fig. 4(b)]. Quantitative comparison of the friction induced deflections for the trace and retrace scans can be obtained from the deflection difference curve shown in Fig. 5(b). The center part of the curve varies around zero, and the absolute value of the right part is nearly the same as the left part defined by $A_{T} C_{T}$. This is because all the forces applied from the surface were indirectly acting upon the cantilever through the flexible MWNT tip. Regardless of the direction of friction, the tensile stress induced by the friction always bends the cantilever toward the substrate. The cantilever is insensitive to the lateral component of the tensile stress since its sensitivity to the buckling force $1 / k_{x}(x$ direction) is only $12 \%$ of its sensitivity to normal force $1 / k_{z}(z$ direction) as estimated using the expression $3 H / 2 L,{ }^{48}$ where $H$ and $L$ are the cantilever tip height and the cantilever length, respectively. The deflection caused by the vertical component of the tensile stress can be calculated by using

$$
D=\frac{F_{F} \cot \theta}{k_{z}}
$$

where $F_{F}$ is the frictional force between the MWNT tip and the tube support layer and the tilting angle of the MWNT with respect to $z$ direction is assumed to be $\theta$ during sliding. The result is not dependent on the scan directions. By using Eq. (3), an estimation of the friction between the MWNT tip and the tube support layer of $0.8 \pm 0.3 \mathrm{nN}$ is obtained from the cantilever deflection of $0.6 \pm 0.2 \mathrm{~nm}$.

\section{B. Scan of multiwalled carbon nanotube tip across suspended single-walled carbon nanotube}

The stripped MWNT tip was flexible enough to scan across the suspended SWNTs without destructive damage to both nanotubes, allowing us to explore the friction between nanotubes. The reason why the stripped MWNT tip can scan across a SWNT is explained below by analyzing the deformation of the MWNT tip during the encounter with the SWNT. When the MWNT tip was advanced into contact with the suspended SWNT, it applied a repulsive force $F$ balanced by the tension of the SWNT. The force increased with the displacement of the tip. By treating the SWNT as an elastic string, the force can be related to the strain of SWNT by (derived from the equations presented by Walters et al $^{44}$ )

$$
F=\frac{2 E A \varepsilon \sqrt{(1+\varepsilon)^{2}-1}}{1+\varepsilon},
$$

where $A$ and $\varepsilon$ are the cross-sectional area and the strain of SWNT (the average diameter of SWNT is $1.43 \mathrm{~nm}^{40}$ ). Assuming a maximum strain of $5.8 \%$ for the SWNT, ${ }^{44}$ the repulsive force $F$ is calculated to be $57.9 \mathrm{nN}$ with this strain. Under this force, a solid MWNT tip $(3.28 \mathrm{~N} / \mathrm{m})$ would be 
bent by $17.7 \mathrm{~nm}$, while a single-shell MWNT tip $(88.3 \mathrm{mN} / \mathrm{m})$ would be bent by $656 \mathrm{~nm}$. The bending of the MWNT tip results in the retraction of the MWNT tip end along $z$ direction of $0.1 \mathrm{~nm}$ and $136 \mathrm{~nm}$ for solid MWNT and single-shell MWNT, respectively. The significantly increased retracting distance of MWNT tip end for single-shell MWNT indicates that after stripping, the MWNT tip could become flexible enough to scan across the SWNT without damaging it.

As shown in Fig. 4, inside the trench, the presence of suspended SWNT is obvious, and it interacted with the MWNT tip when it scanned across the SWNT, reducing cantilever amplitude and causing cantilever deflection. The interaction between the two nanotubes created a band of amplitude attenuation. The band width measured from the profile of representative scan line is around $758 \mathrm{~nm}$ [Fig. 4(a) and the magnified version in Fig. 5(a)]. The variation of the band width is small along the SWNT, reflecting the repetitive nature of the nanotube interaction. The shape of the band is bizarre, with three apparent segments, which was believed to be the consequence of the $X$-branched-morphology of SWNT, also popular in Fig. 1. When the scan direction reversed from trace to retrace, the bands moved to the opposite side of the SWNT. This allows us to subtract the retrace signals from the trace signals to eliminate the noisy background without disrupting useful information for the scan lines across the SWNT. Note that due to the branching of the SWNT, the signals from different scan directions overlap with each other for the scan lines across the SWNT branches. This causes artifacts in the difference images. This problem is circumvented by selecting the representative scan line from the center segment of SWNT, where no side branches are visible.

A close inspection of the tapping amplitude difference curve shown in Fig. 5(a) reveals two subregions $\left(A_{N}-B_{N}\right.$ and $B_{N^{-}} C_{N}$ ) for each band of amplitude attenuation. Here, we use the retrace band as an example, which is reflected upward due to the subtraction (increase in curve means decrease in real signal). At the first region defined by $A_{N}-B_{N}$, the cantilever amplitude was first rapidly reduced by $0.7 \mathrm{~nm}$ followed by slow linear decrease. The amplitude attenuation accelerated at the second region defined by $B_{N^{-}} C_{N}$ (third order was verified to produce a reasonable fit for the nonlinear amplitude curve) to a maximum value of $4.5 \mathrm{~nm}$ and then suddenly the amplitude was restored to the free amplitude (in the difference image, it is zero). In region $A_{N}-B_{N}$, the cantilever deflection showed no difference to the thermal noise. The thermally induced mean cantilever deflection was estimated to be $63 \mathrm{pm}$ using the formula $4 k_{B} T / 3 k_{z},{ }^{49}$ where $k_{B}$ and $T$ are Boltzmann's constant and temperature. It prohibited the detection of forces below $85 \mathrm{pN}$ from static cantilever deflection. Upon entering region $B_{N^{-}} C_{N}$, the cantilever deflection started to monotonically decrease and reach the minimum of $0.5 \mathrm{~nm}$ at point $C_{N}$, then suddenly jumped to zero.

A feasible explanation for the two-phase interaction between nanotubes is [Fig. 5(a)]: First, the MWNT tip came into contact with the SWNT and formed a movable nanotube junction at point $A_{N}$. Due to the frictional force between the nanotubes $\left[F_{F}\right.$ in Fig. $\left.5(\mathrm{a})\right]$, the vibrational energy of the cantilever was partially dissipated, reducing the cantilever amplitude accordingly. When the MWNT tip advanced further with the displacement of the scanner in the $x$ direction, it began to apply increasing repulsive force onto the SWNT $\left[F_{N}\right.$ in Fig. 5(a)]. Due to the low bending stiffness of the MWNT tip, the repulsive force was small, as no remarkable change to cantilever deflection was detected in region $A_{N}-B_{N}$. However, the cantilever amplitude is much more sensitive due to the averaging of cantilever deflection signals over many oscillation cycles, which significantly improves the signal-to-noise ratio. A slight decrease of cantilever amplitude was observed in region $A_{N}-B_{N}$, indicating the friction between the nanotubes was increasing due to the repulsive force between the nanotubes. The MWNT tip and the SWNT continuously deformed with the displacement of the MWNT probe and the nanotube junction slipped toward to the end of the MWNT tip. The shifting of the nanotube junction from the shell of the MWNT tip to its end turned the repulsive force between nanotubes into attraction $\left[F_{A}\right.$ in Fig. 5(a) $]$ at point $B_{N}$. The nature of force changed; however, its magnitude and direction were maintained because the deformations of both nanotubes should not be strongly affected by the shifting of the junction location. The MWNT-end-SWNTshell contact geometry persisted until the final detachment of nanotubes; thus, in region $B_{N^{-}} C_{N}$, the deformation of the nanotubes and the attractive force would increase continuously and smoothly with the displacement of the MWNT probe. At point $C_{N}$, the spring-restoring force of nanotubes exceeded $F_{A}$, and the nanotubes detached from each other. The cantilever deflection measured here should reflect the magnitude of adhesion between the nanotubes.

The width of $A_{N^{-}} C_{N}$ is a measure of the total deformation of the nanotubes in the $x$ direction before they detached from each other. It is about $168 \mathrm{~nm}$ longer than the distance of $A_{T}-B_{T}$, which is a measure of the deformation of the MWNT tip only. If the difference is attributed to the deformation of SWNT (corresponding to a strain of $1.4 \%$ for the SWNT), by comparing it to the deformation of MWNT tip of about $590 \mathrm{~nm}$ (the distance of $A_{T}-B_{T}$ ), it is evident that the bending of MWNT tip is the primary contributor to the band width of amplitude attenuation, confirming that the flexibility of the stripped MWNT tip is very high. In reality, the strain of SWNT should be smaller than $1.4 \%$ since the SWNT could deform out of the $x-y$ plane in compliance with the inclination of MWNT tip (if the SWNT deformed toward the MWNT tip base, a strain of $0.7 \%$ was obtained).

The asymmetrical arrangement of the MWNT tip due to its inclination with respect to the $z$ direction produced certain differences on the trace and retrace scans. When the tip scanned in the retrace direction on the tube support layer, the amplitude was reduced to $5.4 \pm 2.9 \mathrm{~nm}$ as measured from the profile shown in Fig. 4(a), which is about $3 \mathrm{~nm}$ smaller than its trace counterpart. Moreover, the band of amplitude attenuation in the retrace direction is apparently wider than that in the trace direction [Fig. 4(a)]. These evidences suggest the MWNT tip is tilted toward the left side of the probe. However, due to the high flexibility of the MWNT tip, its asymmetrical arrangement has only limited effect on the measured forces. Nearly all scan lines across the SWNT in Fig. 4 ( $\sim 200$ scan lines excluding the few close to the trench 
Tapping amplitude (Trace - Retrace) Tip end at the same height as tube support layer
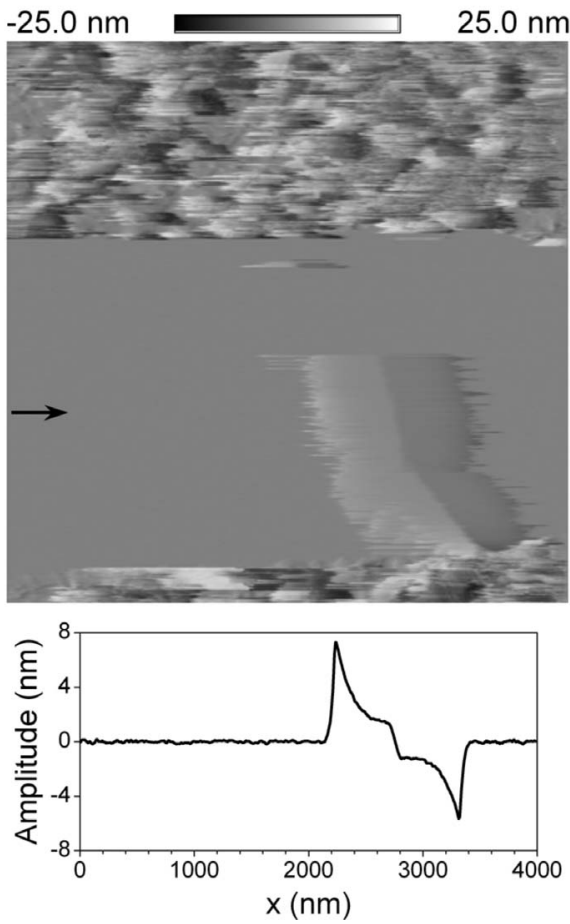

FIG. 6. Cantilever tapping amplitude (trace-retrace) calculated from the captured images with the tip end at the same height as the tube support layer. The profile is given for the scan line marked by the arrow.

wall) were statistically analyzed: for trace signals, the amplitude attenuation measured at points $A_{N}$ and $C_{N}$ are $0.7 \pm 0.1$ and $5.2 \pm 1.5 \mathrm{~nm}$. The deflection measured at point $C_{N}$ is $0.5 \pm 0.2 \mathrm{~nm}$. For retrace signals, the amplitude attenuation measured at points $A_{N}$ and $C_{N}$ are $0.7 \pm 0.1$ and $4.5 \pm 1.4 \mathrm{~nm}$. The deflection measured at point $C_{N}$ is $0.4 \pm 0.2 \mathrm{~nm}$. It appears that the attenuation of trace amplitude and the trace deflection are slightly larger than the retrace counterparts. However, the slight differences remain in the error range.

\section{Scan of multiwalled carbon nanotube on top of single-walled carbon nanotube}

Scans were also performed with the MWNT tip end nearly at the same height as the SWNT and the tube support layer. The amplitude difference image is shown in Fig. 6. The upper part of the SWNT was unable to contact with the MWNT tip, indicating the sample surface is slightly tilted down at the top side. Note that at the scan line where the MWNT tip first picked up the SWNT, a band of amplitude attenuation immediately emerged, and its width was kept to be nearly constant for the following scan lines. Different from the above observation when the tip end is $100 \mathrm{~nm}$ below the SWNT, the interaction here is expected to immediately establish on the MWNT-end-SWNT-shell contact geometry, bypassing the $A_{N^{-}} B_{N}$ part where the MWNT connected to the SWNT with its outer shell. The average bandwidth measured from the profile in Fig. 6 is $539 \mathrm{~nm}$, which is $219 \mathrm{~nm}$ shorter than that measured from Fig. 5(a). If $B_{N}$ is defined by linearly extrapolating the deflection slope to zero deflection, the difference is close to the distance of $A_{N^{-}} B_{N}$ of $293 \mathrm{~nm}$ measured in Fig. 5(a), suggesting that region $A_{N}-B_{N}$ is not present in Fig. 6 . The statistical analysis of Fig. 6 yields the following: for trace signals, the amplitude attenuations measured during the initial contact and the peak are $1.5 \pm 0.3$ and $4.8 \pm 1.2 \mathrm{~nm}$. For retrace signals, they are $1.7 \pm 0.3$ and $6.2 \pm 1.2 \mathrm{~nm}$, respectively. Compared to the results obtained from Fig. 4, the maximum amplitude attenuation is increased by only $0.7 \mathrm{~nm}$ (still within the statistical distribution), while the average amplitude attenuation during the initial contact is significantly increased by $0.9 \mathrm{~nm}$ (a $130 \%$ increase). Such difference distinguishes point $B_{N}$ from $A_{N}$ in terms of nanotube contact geometry, confirming the division of the band of amplitude attenuation in Figs. 4 and 5(a) into two subregions, in which the MWNT tip connected to the SWNT using its shell and its end, respectively.

\section{Adhesion between nanotubes}

The adhesion between nanotubes was evaluated from the cantilever deflection at the point where the nanotubes detached from each other $\left(C_{N}\right)$. By multiplying $k_{z}$ with the average cantilever deflection of $0.5 \pm 0.2 \mathrm{~nm}$ obtained before, the adhesion between nanotubes is evaluated to be $0.7 \pm 0.3 \mathrm{nN}$. This value should be the low-bound estimation of the adhesive force since the tilting angle of the MWNT tip at the junction is unknown. Using the Johnson-KendallRoberts (JKR) model $^{50}$ and assuming the MWNT has a diameter of $75 \mathrm{~nm}$ as stripped from the 100-nm-diameter MWNT and the SWNT has a diameter of $1.43 \mathrm{~nm}$, the work of adhesion between nanotubes was $0.03 \mathrm{~N} / \mathrm{m}$ as estimated by using

$$
W_{A}=\frac{2 F_{A}}{3 \pi \sqrt{r_{1} r_{2}}},
$$

where $r_{1}$ and $r_{2}$ are the radii of the MWNT tip and the SWNT, respectively. This value is about ten times smaller than that derived from reported surface energy of graphite. ${ }^{51,52}$ The primary errors could come from the determination of $F_{A}$ and the use of $37.5 \mathrm{~nm}$ as $r_{1}$. At the point of detachment, the MWNT tip connected to the SWNT with its end, and the effective radius of curvature at the MWNT end could be much smaller than the radius of the MWNT.

\section{E. Friction between nanotubes}

To evaluate the frictional force between nanotubes, the amplitude attenuation during the initial contact of nanotubes is employed. The vibrating cantilever needs to be modeled as a harmonic oscillator with external driving and damping. ${ }^{53} \mathrm{In}$ equilibrium, the average input of power by the external driver $\bar{P}_{\text {in }}$ must equal the average power dissipated by the motion of the cantilever beam $\bar{P}_{0}$ and by the tip-sample interaction $\bar{P}_{\text {tip }}$, 


$$
\bar{P}_{\text {in }}=\bar{P}_{0}+\bar{P}_{\text {tip }} .
$$

In order to determine $\bar{P}_{\text {tip }}$, the other two terms, $\bar{P}_{\text {in }}$ and $\bar{P}_{0}$, have first to be measured and calculated. Assuming the driving frequency is chosen to be the natural resonance frequency of cantilever $\omega_{0}$, by integrating the instantaneous power fed into the dynamic system over one oscillation period, $\bar{P}_{\text {in }}$ was calculated to be ${ }^{53}$

$$
\bar{P}_{\text {in }}=\frac{k_{z} \omega_{0} A_{0} A \sin \varphi}{2 Q},
$$

where $A_{0}$ and $A$ are unloaded amplitude and loaded amplitude (nanotubes are in contact), and $\varphi$ and $Q$ are phase shift and quality factor. The damping source $\bar{P}_{0}$ contains two components: the intrinsic damping caused by the movement of the cantilever's tip relative to its base and the viscous damping from air. Similarly, by integrating the instantaneous power dissipation over one oscillation period, $\bar{P}_{0}$ was expressed as ${ }^{53}$

$$
\bar{P}_{0}=\frac{k_{z} \omega_{0} A^{2}}{2 Q} .
$$

If the frictional force between nanotubes does not depend on the velocity of the MWNT tip, the average power dissipated by the friction can be simply written as

$$
\bar{P}_{\text {tip }}=2 F_{F} \omega_{0} A / \pi \text {. }
$$

Substituting Eqs. (7)-(9) into Eq. (6), friction is related to the amplitude attenuation by

$$
F_{F}=\frac{\pi k_{z}\left(A_{0} \sin \varphi-A\right)}{4 Q} .
$$

Using the average amplitude attenuation of $0.7 \pm 0.1 \mathrm{~nm}$ obtained before as $A_{0}-A$, with a measured quality factor of 165 , a value of $4 \pm 1 \mathrm{pN}$ is calculated for friction between nanotubes without the knowledge of the phase shift. By dividing the friction with the adhesion measured before, an upper limit of $0.006 \pm 0.003$ is obtained as the experimental value of coefficient of friction between nanotubes in ambient. The value is an upper bound because the friction is overestimated, and the adhesion between nanotube shells is underestimated as discussed before. This value is comparable to the reported value of graphite on the nanoscale. ${ }^{21,54}$ It was reported that the primary mechanism of friction in graphite is atomic-scale stick slip.

The shear strength between nanotubes is estimated by using the JKR model. ${ }^{50}$ The contact area between nanotubes at zero force is given by

$$
A=\pi\left(6 \pi W_{A} r_{1} r_{2} / K\right)^{2 / 3},
$$

where $K$ is the composite Young's modulus, given as $K$ $=2 E / 3\left(1-\nu^{2}\right)$, where $\nu$ is the Poisson ratio of nanotube, which is assumed to be 0.3 . By using a reported value of $0.22 \mathrm{~N} / \mathrm{m}$ as the work of adhesion between nanotubes, ${ }^{51}$ we obtain a contact area at zero force of $0.9 \mathrm{~nm}^{2}$. The shear strength obtained by dividing the friction with the contact area is $4 \pm 1 \mathrm{MPa}$, which is close to the value of $2 \mathrm{MPa}$ obtained by sliding MWNT on graphite in ambient. ${ }^{39}$ Our result confirms that the intertube shear strength is about 2 orders of magnitude larger than the interlayer shear strength for defect-free nanotubes. There are two possible reasons for the increased intertube shear strength in ambient. First, in our experimental conditions with a relative humidity of $30 \pm 5 \%$, a monolayer of water might be present at the nanotube interface due to physical adsorption and/or capillary condensation, altering the friction property of the nanotube junction. Recent theoretical simulations on confined water between graphene sheets support this hypothesis. ${ }^{55-57}$ A monolayer of water can condense into the slit by the joint stabilizing effect of the opposing graphite surfaces. An estimation of the static friction coefficient using a wide range of water-graphite binding energy yields values within $0.001-0.015$, coinciding with our experimental result of $0.006 \pm 0.003$. In contrast, the interlayer shear strength was measured in vacuum, free of all the adventitious species. Also, the small interlayer distance of $0.34 \mathrm{~nm}$ can prevent other molecules from entering between the shells of MWNT. ${ }^{24,26}$ Second, the continuum model might not be accurate enough for the calculation of the contact area between nanotubes. The SWNT might bend and comply with the circumference of MWNT tip to increase the contact area. To further clarify this possibility, more works based on molecular mechanics are certainly favored. ${ }^{58}$

Although there are a few spikes in region $A_{N}-B_{N}$ on the amplitude curve shown in Fig. 5(a) when the MWNT tip slid on the SWNT, they are more likely to arise from random defects presenting on the MWNT tip rather than the regular stick-slip occurred at atomic scale at the nanotube-nanotube interface. In principle, the incommensurate contact between tube pairs should be able to suppress the collective stick-slip motion (as in the nanotube-graphite sliding friction). Only with commensurate contact, as observed in the nanotubegraphite rolling friction, may pronounced atomic scale stickslip occur. The presence of defects may also introduce an additional energy dissipating mechanism, causing stick-slip in the interlayer sliding friction. In our system, the sliding situation is different from the sliding between nanotube and graphite, where the contact interface fully extended to the entire length of the nanotube. The MWNT tip and the SWNT contacted at crossed geometry, and the contact area between them is small (composed of $\sim 35$ carbon atoms), possibly allowing the distinguishing of a small potential difference along the MWNT if the signal-to-noise ratio is high enough. However, in our experimental setup, the vibrational amplitude $(\sim 20 \mathrm{~nm})$, leading to an averaging of interaction across $\sim 286$ carbon atoms during one oscillating cycle, is too large for observing atomic scale friction behavior. In order to observe it, using an extremely small cantilever amplitude (on the order of $0.1 \mathrm{~nm}$ ) with lock-in amplifier might be a feasible choice. ${ }^{59}$ Such observation is expected to reveal more details regarding the atomic scale friction mechanism between nanotubes as well as the defect distribution on the MWNT.

\section{SUMMARY}

In this paper, we reported a method to characterize the adhesion and friction properties between individual carbon 
nanotubes by using the tapping mode AFM in ambient. A MWNT tip attached to a conventional AFM probe was scanned across a SWNT suspended on a microtrench at crossed geometry by using the area scan function of AFM. The adhesion and friction between nanotubes was evaluated from the AFM cantilever response during scanning. The MWNT tip used was extremely compliant to be able to scan across the suspended SWNT and on the tube support layer without destructive damage to both nanotubes. Experimental evidence such as SEM images and force-distance curves suggests that the MWNT tip had a thin tip with a length of $\sim 880 \mathrm{~nm}$ extending from a thick base, formed possibly by stripping part of the outer shells from the original MWNT tip due to so-called sword-in-sheath breaking mechanism. The tip model was found to provide satisfying explanations on the scanning behavior of the MWNT tip on the tube support layer.

Area scans covering suspended SWNTs were performed with the MWNT tip end at different heights with respect to the tube support layer by disabling the AFM feedback response. The interaction of the MWNT tip with the SWNTs during area scans generated bands of tapping amplitude attenuation in the AFM amplitude images. For the area scan with the MWNT tip end $\sim 100 \mathrm{~nm}$ below the tube support layer, each band of amplitude attenuation was composed of two subregions: one was formed during the sliding of the MWNT tip shell on the SWNT shell (the sliding region) and the other one was formed during the detachment of the MWNT tip end from the SWNT shell (the detaching region). For the area scan with the MWNT tip end at the same height as the tube support layer, only the second region, the detaching region, was found as the contact between nanotubes was established immediately on the MWNT tip end with the SWNT shell.
In the sliding region, a quantitative relation between the sliding friction between nanotubes and the cantilever amplitude attenuation was derived based on the analysis of the dissipated power of cantilever vibration. The sliding friction between nanotubes was calculated to be $4 \pm 1 \mathrm{pN}$ from the cantilever tapping amplitude attenuation of $0.7 \pm 0.1 \mathrm{~nm}$ summarized over $\sim 200$ scan lines across the SWNT. In the detaching region, the adhesion between nanotubes was calculated to be $0.7 \pm 0.3 \mathrm{nN}$ from the maximum cantilever deflection of $0.5 \pm 0.2 \mathrm{~nm}$ at the detachment of nanotubes. From the adhesion and friction, an experimental value of coefficient of friction between nanotubes was calculated to be about $0.006 \pm 0.003$, which is comparable to the reported value of graphite on nanoscale. The shear strength between nanotubes was estimated to be $4 \pm 1 \mathrm{MPa}$ by using the JKR model, in accordance with the value of $2 \mathrm{MPa}$ reported for the sliding of MWNT on graphite in ambient. It is nearly 2 orders of magnitude larger than the reported interlayer shear strength for defect-free MWNTs in vacuum. We attribute this difference to the presence of water at the nanotube-nanotube interface, altering the friction property between nanotubes. The ability to form, modulate, and characterize the intertube properties (including but not limited to the adhesion and friction properties) at the single nanotube level by using the present method may find broad applications with functionalized nanotubes and nanotube junctions.

\section{ACKNOWLEDGMENTS}

The authors gratefully acknowledge Cattien V. Nguyen for providing the MWNT probe, Kwang Joo Kwak for SEM measurements, and ETH Zurich's FIRST team for cleanroom support. Funding support from the Swiss National Science Foundation (Grant No. 200021-108059/1) is greatly acknowledged.
*Author to whom correspondence should be addressed; bhushan.2@osu.edu

${ }^{1}$ S. Iijima, Nature (London) 354, 56 (1991).

${ }^{2}$ M. S. Dresselhaus, G. Dresselhaus, and P. Avouris, Carbon Nanotubes: Synthesis, Structure, Properties, and Applications (Springer, New York, 2000).

${ }^{3}$ B. Bhushan, Springer Handbook of Nanotechnology (Springer, Berlin, 2007).

${ }^{4}$ D. H. Robertson, D. W. Brenner, and J. W. Mintmire, Phys. Rev. B 45, 12592 (1992).

${ }^{5}$ J. P. Salvetat, G. A. D. Briggs, J. M. Bonard, R. R. Bacsa, A. J. Kulik, T. Stockli, N. A. Burnham, and L. Forro, Phys. Rev. Lett. 82, 944 (1999).

${ }^{6}$ M. M. J. Treacy, T. W. Ebbesen, and J. M. Gibson, Nature (London) 381, 678 (1996).

${ }^{7}$ E. W. Wong, P. E. Sheehan, and C. M. Lieber, Science 277, 1971 (1997).

${ }^{8}$ M. F. Yu, O. Lourie, M. J. Dyer, K. Moloni, T. F. Kelly, and R. S. Ruoff, Science 287, 637 (2000).

${ }^{9}$ M. R. Falvo, G. J. Clary, R. M. Taylor, V. Chi, F. P. Brooks, S. Washburn, and R. Superfine, Nature (London) 389, 582 (1997).
${ }^{10}$ B. I. Yakobson, C. J. Brabec, and J. Bernholc, Phys. Rev. Lett. 76, 2511 (1996).

${ }^{11}$ J. W. G. Wildoer, L. C. Venema, A. G. Rinzler, R. E. Smalley, and C. Dekker, Nature (London) 391, 59 (1998).

${ }^{12}$ E. T. Thostenson, Z. F. Ren, and T. W. Chou, Compos. Sci. Technol. 61, 1899 (2001).

${ }^{13}$ H. J. Dai, J. H. Hafner, A. G. Rinzler, D. T. Colbert, and R. E. Smalley, Nature (London) 384, 147 (1996).

${ }^{14}$ S. S. Wong, E. Joselevich, A. T. Woolley, C. L. Cheung, and C. M. Lieber, Nature (London) 394, 52 (1998).

${ }^{15}$ R. H. Baughman, C. X. Cui, A. A. Zakhidov, Z. Iqbal, J. N. Barisci, G. M. Spinks, G. G. Wallace, A. Mazzoldi, D. De Rossi, A. G. Rinzler, O. Jaschinski, S. Roth, and M. Kertesz, Science 284, 1340 (1999).

${ }^{16}$ P. Kim and C. M. Lieber, Science 286, 2148 (1999).

${ }^{17}$ R. H. Baughman, A. A. Zakhidov, and W. A. de Heer, Science 297, 787 (2002).

${ }^{18}$ P. C. Collins, M. S. Arnold, and P. Avouris, Science 292, 706 (2001).

${ }^{19}$ T. Rueckes, K. Kim, E. Joselevich, G. Y. Tseng, C. L. Cheung, and C. M. Lieber, Science 289, 94 (2000). 
${ }^{20}$ S. J. Tans, A. R. M. Verschueren, and C. Dekker, Nature (London) 393, 49 (1998).

${ }^{21}$ B. Bhushan, J. N. Israelachvili, and U. Landman, Nature (London) 374, 607 (1995).

${ }^{22}$ K. T. Lau and D. Hui, Carbon 40, 1605 (2002).

${ }^{23}$ B. Bhushan, B. Galasso, C. Bignardi, C. V. Nguyen, L. Dai, and L. Qu, Nanotechnology 19, 125702 (2008).

${ }^{24}$ J. Cumings and A. Zettl, Science 289, 602 (2000).

${ }^{25}$ A. M. Fennimore, T. D. Yuzvinsky, W. Q. Han, M. S. Fuhrer, J. Cumings, and A. Zettl, Nature (London) 424, 408 (2003).

${ }^{26}$ A. Kis, K. Jensen, S. Aloni, W. Mickelson, and A. Zettl, Phys. Rev. Lett. 97, 025501 (2006).

${ }^{27}$ A. N. Kolmogorov and V. H. Crespi, Phys. Rev. Lett. 85, 4727 (2000).

${ }^{28}$ J. Servantie and P. Gaspard, Phys. Rev. Lett. 91, 185503 (2003).

${ }^{29}$ M. F. Yu, B. I. Yakobson, and R. S. Ruoff, J. Phys. Chem. B 104, 8764 (2000).

${ }^{30}$ Y. Zhao, C. C. Ma, G. H. Chen, and Q. Jiang, Phys. Rev. Lett. 91, 175504 (2003).

${ }^{31}$ M. Dienwiebel, G. S. Verhoeven, N. Pradeep, J. W. M. Frenken, J. A. Heimberg, and H. W. Zandbergen, Phys. Rev. Lett. 92, 126101 (2004).

${ }^{32}$ M. Hirano and K. Shinjo, Phys. Rev. B 41, 11837 (1990).

${ }^{33}$ P. Tangney, S. G. Louie, and M. L. Cohen, Phys. Rev. Lett. 93, 065503 (2004).

${ }^{34}$ F. P. Bowden and D. Tabor, The Friction and Lubrication of Solids (Clarendon, Oxford, 1950).

${ }^{35}$ B. Bhushan, Nanotribology and Nanomechanics - an Introduction (Springer, Berlin, 2005).

${ }^{36}$ B. Bhushan, Introduction to Tribology (Wiley, New York, 2002).

${ }^{37}$ M. R. Falvo, J. Steele, R. M. Taylor, and R. Superfine, Tribol. Lett. 9, 73 (2000).

${ }^{38}$ M. R. Falvo, J. Steele, R. M. Taylor, and R. Superfine, Phys. Rev. B 62, R10665 (2000).

${ }^{39}$ M. R. Falvo, R. M. Taylor, A. Helser, V. Chi, F. P. Brooks, S. Washburn, and R. Superfine, Nature (London) 397, 236 (1999).

${ }^{40}$ A. Jungen, S. Hofmann, J. C. Meyer, C. Stampfer, S. Roth, J.
Robertson, and C. Hierold, J. Micromech. Microeng. 17, 603 (2007).

${ }^{41}$ A. Jungen, C. Stampfer, J. Hoetzel, V. M. Bright, and C. Hierold, Sens. Actuators, A 130, 588 (2006).

${ }^{42}$ C. V. Nguyen, Q. Ye, and M. Meyyappan, Meas. Sci. Technol. 16, 2138 (2005).

${ }^{43}$ J. E. Sader, J. W. M. Chon, and P. Mulvaney, Rev. Sci. Instrum. 70, 3967 (1999).

${ }^{44}$ D. A. Walters, L. M. Ericson, M. J. Casavant, J. Liu, D. T. Colbert, K. A. Smith, and R. E. Smalley, Appl. Phys. Lett. 74, 3803 (1999).

${ }^{45}$ H. J. Butt, B. Cappella, and M. Kappl, Surf. Sci. Rep. 59, 1 (2005).

${ }^{46}$ R. J. Roark, W. C. Young, and R. G. Budynas, Roark's Formulas for Stress and Strain (McGraw-Hill, New York, 2002).

${ }^{47}$ H. W. Yap, R. S. Lakes, and R. W. Carpick, Nano Lett. 7, 1149 (2007).

${ }^{48}$ D. F. Ogletree, R. W. Carpick, and M. Salmeron, Rev. Sci. Instrum. 67, 3298 (1996).

${ }^{49}$ H. J. Butt and M. Jaschke, Nanotechnology 6, 1 (1995).

${ }^{50}$ K. L. Johnson, K. Kendall, and A. D. Roberts, Proc. R. Soc. London, Ser. A 324, 301 (1971).

${ }^{51}$ L. X. Benedict, N. G. Chopra, M. L. Cohen, A. Zettl, S. G. Louie, and V. H. Crespi, Chem. Phys. Lett. 286, 490 (1998).

${ }^{52}$ R. J. Good, L. A. Girifalco, and G. Kraus, J. Phys. Chem. 62, 1418 (1958).

${ }^{53}$ J. P. Cleveland, B. Anczykowski, A. E. Schmid, and V. B. Elings, Appl. Phys. Lett. 72, 2613 (1998).

${ }^{54}$ J. A. Ruan and B. Bhushan, J. Appl. Phys. 76, 5022 (1994).

${ }^{55}$ A. Pertsin and M. Grunze, J. Phys. Chem. B 108, 1357 (2004).

${ }^{56}$ J. C. Liu and P. A. Monson, Langmuir 21, 10219 (2005).

${ }^{57}$ A. Pertsin and M. Grunze, J. Chem. Phys. 125, 114707 (2006).

${ }^{58}$ T. Hertel, R. E. Walkup, and P. Avouris, Phys. Rev. B 58, 13870 (1998).

${ }^{59}$ A. Maali, T. Cohen-Bouhacina, G. Couturier, and J. P. Aime, Phys. Rev. Lett. 96, 086105 (2006). 